structures, for example, on

chromosome 8 .

The authors identified two sets of substantial inter-chromosomal interactions, or 'hubs', corresponding to gene-poor, transcriptionally inactive regions or to gene-dense regions enriched for features of transcriptional activity. Subsequent characterization of these hubs by mapping RNA and DNA interactions simultaneously showed that the inactive hub regions organized around the nucleolus, whereas interactions between regions in the active hub were organized around nuclear speckles, which contain proteins involved in mRNA splicing and processing.

Taken together, the findings by Quinodoz et al. suggest that nuclear bodies act as interaction hubs between chromosomes to modulate genome organization within the nucleus.

ORIGINAL ARTICLE Quinodoz, S. A. et al. Higher-order inter-chromosomal hubs shape 3D genome organization in the nucleus. Cell. https://doi.org/10.1016/j.cell.2018.05.024 (2018) FURTHER READING Bonev, B. \& Cavalli, G. et al. Organization and function of the $3 \mathrm{D}$ genome. Nat. Rev. Genet. 17, 661-678 (2016)

a database of variants that have been classified for clinical significance based on their presence in human disease, and found that mutations changing the constrained sites were over-represented in deleterious categories. Both lines of evidence point to these constraints as being important in human disease, and further examination of specific mutations in cancer, Alzheimer disease and neurodevelopmental disorders suggested their impact is broad.

While constraint at sequences around splice sites has been posited since the donor and acceptor sites were first defined, an understanding of their role in human disease is lacking. This study provides specificity for clinical exome analysis, although broader application and metrics await larger data sets.

Rebecca Furlong, Senior Editor, Nature Communications

ORIGINAL ARTICLE Zhang, S. et al.

Base-specific mutational intolerance near splice-sites clarifies role of non-essential splice

nucleotides. Genome Res. https://do

org/10.1101/gr.231902.117 (2018)

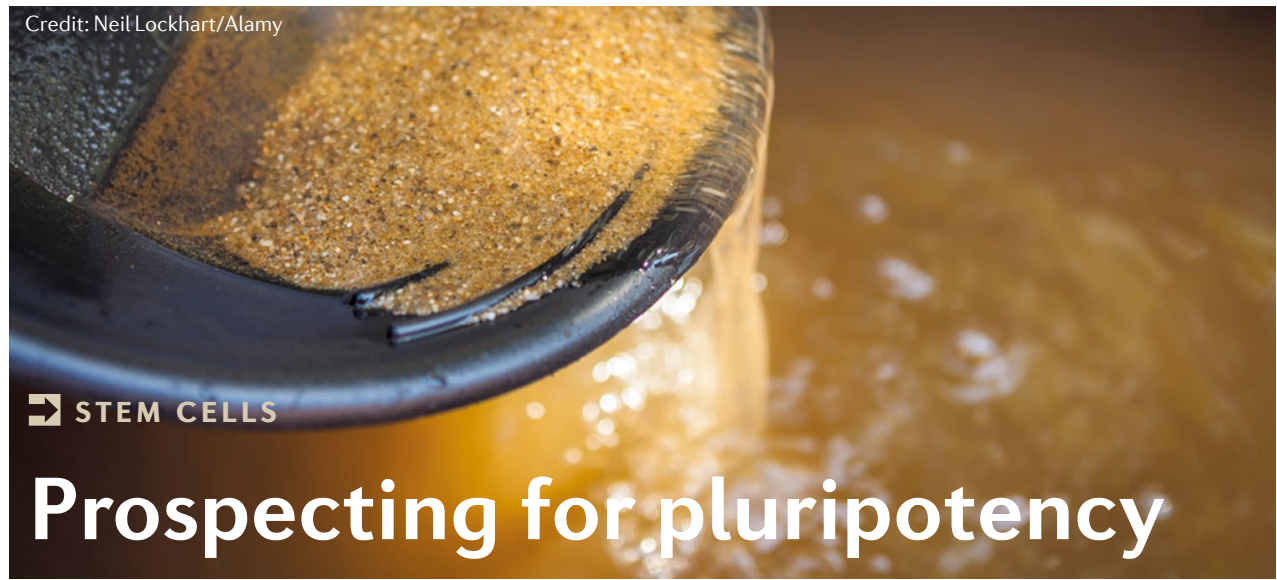

Planaria are flatworms that can regenerate their entire body from a tiny tissue fragment. This remarkable property is mediated by neoblasts, highly heterogeneous populations of proliferating cells. Neoblasts are unusual because they include adult pluripotent stem cells (PSCs) - most organisms do not maintain PSCs beyond embryogenesis. However, characterization of these adult PSCs has been hampered by the inability to discriminate them from other neoblast subtypes. Now, a study in Cell reports the prospective identification and isolation of a neoblast subpopulation containing adult PSCs, the characterization of which should help determine the mechanisms underlying pluripotency and tissue regeneration.

Previous studies isolated a neoblast subpopulation enriched in PSCs (termed X1) from dissociated animal cells by fluorescence-activated cell sorting (FACS), but it contained only a small proportion of pluripotent cells capable of fully rescuing sublethally irradiated animals, which were termed clonogenic neoblasts (cNeoblasts). Other studies identified three classes of neoblasts $(\sigma, \zeta$ and $\gamma)$ based on basic gene expression and functional profiles. The $\sigma$-class display some stem cell-like behaviour but, because they are defined empirically, they cannot be prospectively identified and isolated to determine if they are homogenous populations of $\mathrm{cNeoblasts.}$

Zeng et al. set out to overcome this problem by defining prospective molecular signatures for different neoblast subpopulations. Neoblasts are known to express piwi-1, but the authors observed that levels of piwi-1 RNA varied between cells. Using FACS, they separated neoblasts according to their levels of piwi-1 RNA and PIWI-1 protein expression and showed that cells expressing the highest levels of both piwi-1 RNA and PIWI-1 protein (Piwi-1 ${ }^{\text {High }}$ ) have characteristics of PSCs. However, similar to X1, only some Piwi-1 ${ }^{\text {High }}$ cells were pluripotent. Furthermore, they are transcriptionally distinct from $\mathrm{X} 1$ and $\sigma$-class cells, suggesting each is a different heterogeneous neoblast subpopulation containing cNeoblasts.
To identify molecular signatures representative of different subpopulations, the authors performed single-cell RNA sequencing (scRNA-seq) on 7,614 Piwi- $1^{\text {High }} X 1$ cells. The 7,088 cells that passed quality control clustered into 12 neoblast subpopulations (Nb1-Nb12), and discriminative gene sets were subsequently determined and validated for each cell cluster. The authors defined four criteria to determine which subclass most likely contained PSCs. First, the cell cluster should express high levels of piwi-1, $\sigma$-class gene markers and self-renewal regulator genes. Second, it should not express high levels of progenitor cell marker genes. Third, gene expression should increase after amputation to reflect the observed increase in PSC numbers in response to injury. Finally, it should have a distinct expression profile that reflects the ability of cNeoblast cells to survive sublethal irradiation. Only the Nb2 cluster - defined by expression of the tetraspanin-1 (TSPAN-1) membrane protein - met all four criteria, suggesting it alone contains pluripotent neoblasts. To confirm the stem cell properties of Nb2 cells, TSPAN-1-expressing cells were isolated by FACS for use in single-cell transplantation experiments; $23 \%$ of transplanted $\mathrm{Nb} 2$ cells rescued lethally irradiated animals - a 14-fold improvement over X1 rescue efficiency.

Subsequent transcriptional profiling experiments point to the huge potential afforded by the prospective identification and isolation of neoblast subpopulations. For instance, pluripotent $\mathrm{Nb} 2$ cells have been shown to undergo differential transcriptional responses to homeostasis, irradiation and injury.

Taken together, these studies suggest that in-depth characterization of PSCs and other neoblast subclasses throughout development is now a realistic prospect.

\section{Dorothy Clyde}

ORIGINAL ARTICLE Zeng, A. et al. Prospectively isolated Tetraspanin ${ }^{+}$neoblasts are adult pluripotent stem cells underlying planaria regeneration. Cell 173, 1593-1608 (2018) 